\title{
Short-term postoperative distress associated with open vs. transoral robotic surgery (TORS) in patients with T1-T2 carcinomas of the tongue base and supraglottis
}

\author{
Karel Slama ${ }^{\mathrm{a}}$, David Slouka ${ }^{\mathrm{b}}$, Jaroslav Slipka ${ }^{\mathrm{b}}$, Slavomil Fischer ${ }^{\mathrm{a}}$
}

\begin{abstract}
Background. This study compared the adverse effects of open surgery (OS) including lateral pharyngotomy and supraglottic laryngectomy vs. transoral robotic surgery (TORS) in the treatment of stage T1 and T2 carcinomas of the tongue base and supraglottis.

Methods. A retrospective study involving a 49 (13 female and 36 male) patients with untreated T1 or T2 carcinomas. Twenty two were operated on using TORS and 27 underwent conventional OS. The indicators for comparison were: total blood loss during surgery, post-operative pain measured with the Visual Analog Scale (VAS); global, emotional and physical post-operational states assessed with the standardized M.D. Anderson Dysphagia Inventory (MDADI) and psychosocial distress (PD) questionnaire. Apart from blood loss, subjective symptoms were evaluated 1 and 6 weeks and 6 months after surgery. The differences in indicators between groups were analyzed using Fisher's Least Significant Difference (LSD) test at the $5 \%$ significance level.

Results. Mean general OS and TORS associated blood loss were 405 and $29 \mathrm{ml}$, respectively. The mean MDADI score in TORS vs. OS patients one week, six weeks and six months postoperatively was 60.01 vs. $44.93,91.01$ vs. 62.19 and 94.18 vs. 93.56. The mean VAS score in the TORS vs. OS group at the same time intervals were 5.09 vs. $5.56,2.09$ vs. 3.11 and 1.27 vs. 1.33. All differences between TORS and OS were statistically significant with the exception of 6 month values for particular scores. The mean PD score in TORS vs. OS patients in one week, six weeks and 6 months was 26.82 vs. $25.11,39.95$ vs. 29.22 and 44.73 vs. 44.52 . Only the six week distinctions were significant. The both methods were comparable in terms of the risk of locoregional tumour recurrence.

Conclusions. The study confirmed the assumption of the TORS as a minimally invasive procedure significantly reducing the intraoperative blood loss, pain, swallowing and psychosocial distress as late as 6 weeks postoperatively in patients with early staged carcinomas of the tongue base and supraglottis.
\end{abstract}

Key words: open surgery, transoral robotic surgery, carcinoma of tongue base and supraglottis, blood loss, MDADI, VAS, psychosocial distress

Received: December 12, 2015; Accepted: March 8, 2016; Available online: March 17, 2016

http://dx.doi.org/10.5507/bp.2016.015

${ }^{a}$ Department of Otorhinolaryngology, Head and Neck Surgery Department, Masaryk Hospital in Usti nad Labem and Department of Education, Faculty of Education, J. E. Purkyne University in Usti nad Labem, Czech Republic

${ }^{b}$ Department of Otorhinolaryngology, Head and Neck Surgery Department, Faculty of Medicine in Pilsen, Charles University in Prague, Pilsen, Czech Republic

Corresponding author: Karel Slama, e-mail karel.slama@kzcr.eu

\section{INTRODUCTION}

Squamous cell carcinomas (SCC) of the oropharynx and larynx occur more frequently in males aged over 60 years. In the last decade, an increase in incidence - females included, of these tumours has been recorded ${ }^{1}$. $40-50 \%$ of patients are those with a background of heavy smoking, alcoholism and insufficient mouth cavity hygiene. 50-60\% of oropharyngeal carcinomas are associated with human papillomaviruses HPV ( ref. $^{2}$ ). A dramatic growth in the occurrence of HPV induced carcinoma has been recently observed especially in young, non-smokers ${ }^{3}$. SCCs located in the tongue base and supraglottis are diseases that are poorly accessible due to their anatomic positions.

When an external approach in terms of lateral pharyngotomy and supraglottic partial laryngectomy is used for the removal of the tongue base and supraglottic SCC carcinoma, respectively, it is necessary to disrupt the integrity of the neck structures, starting with the skin down to the pharynx. In the case of a carcinoma in the supraglottis, invasion in cartilaginous structures of the larynx is unavoidable.

In contrast, both transoral robotic surgery (TORS) and transoral laser microsurgery use natural access through the oral cavity and do not damage soft tissues of the neck ${ }^{4,5}$.

The benefits of the latter operation are well known. However, TORS allows an even clearer and wider, 3D view of the surgical field ${ }^{6-9}$.

The present study compares the adverse effects of surgical removal of histologically-proven T1 and T2 SCC of tongue base and supraglottis, performed by two different 
techniques, namely by conventional open surgery (OS) and TORS (ref. ${ }^{10-12}$ ). To the best of our knowledge - such a comparison has not been made so far.

\section{METHODS}

In this retrospective study, a total of 49 patients (13 female and 36 male) were enrolled. The average age of females and males was 61.5 (range 43-78) and 57.6 (range $27-75$ ) years, respectively. The inclusion criteria were the presence of tongue base or supraglottic tumour staged T1 or T2 according to TNM classification ${ }^{13}$. The patients in poor general conditions and those with cervical lymph node metastasis showing radiological signs of invasion in the carotid artery as well as with tumours considered inadvisable for TORS by their CT or MRI characteristics, were excluded ${ }^{14}$.

All TORS procedures were carried out by the leading author at the Otorhinolaryngology, Head and Neck Surgery Department, Masaryk Hospital Usti nad Labem, from September 2011 to November 2014. Seventeen of the 27 OS procedures were accomplished at the Otorhinolaryngology, Head and Neck Surgery Department, Charles University in Prague, Faculty of Medicine in Pilsen. Vinci S-HD Surgical System was applied for robotic operations. The working $5 \mathrm{~mm}$ wristed robotic instruments, inserted through the oral cavity into the difficult to access area of the tongue base and the supraglottis, were completely controlled by the surgeon's movements with the handles inserted in the console, located at a distance from the patient. Open surgery included lateral pharyngotomy to approach tongue base carcinomas or supraglottic partial laryngectomy.

All patients signed written informing consent, elaborated and approved by the review board at the institution of the leading author.

Conventional OS and TORS was performed in 27 (8 females and 19 males) and 22 (5 females and 17 males) patients, respectively. Twelve of tongue base tumours were performed by TORS and by 10 lateral pharyngotomy. Ten and 17 patients diagnosed with supraglottic SCC were operated on using TORS and open supraglottic laryngectomy, respectively.

Elective I-III bilateral neck dissection was performed in all 19 (39\%) cN0 patients. Selective I-V or comprehensive I-VI bilateral dissection was executed in all 30 (61\%) $\mathrm{cN}+$ cases.

All but one tumour, namely cribriform adenocarcinoma of the tongue, were well (11 pts.) moderately (30 pts) and poorly differentiated ( 7 pts.) squamous cell carcinomas.

All patients had nasogastric feeding tube (NGFT) for enteral alimentation postoperatively. This was removed immediately after the patient could tolerate both liquid and soft diet without aspiration. Postoperatively all patients were kept on analgesics (Perfalgan $2 \mathrm{~g}$ a day) for one week. Some had to continue on this therapy but no longer than for 6 further days.
Indications for postoperative radiotherapy or radiochemotherapy followed the recommendations reported by Bernier et al. ${ }^{15}$. Nineteen (39\%) patients were treated solely by surgery. Fourteen cases (28\%) received postoperative radiotherapy alone. Initially, sixteen (33\%) patients, including four with tongue base SCC and twelve with supraglottic SCC, were indicated for adjuvant radiochemotherapy with cis-platin. However, five of them refused chemotherapy and they received radiotherapy only.

The follow-up ranged from 6 to 41 (mean 34) months. There were no locoregional recurrences in $11 / 12$ (92\%) and 10/10 (100\%) tongue base SCC and 9/10 (90\%) and 16/17 (94\%) supraglottic SCC cases after TORS and OS, respectively. Distant metastases occurred in $2 / 12$ (17\%) and $2 / 10(20 \%)$ tongue base SCC and $1 / 10(10 \%)$ and 1/17 (6\%) supraglottic SCC cases after TORS and OS, respectively. One TORS patient who had refused chemotherapy, died of local reccurence of a tongue base SCC, another one succumbed to high malignant pancreatic adenocarcinoma, without signs of recurrent supraglottic carcinoma. One OS patient who had developed concurrent prostatic adenocarcinoma, died of local recurrence of supraglotic SCC.

The study compared the following data obtained from both surgical techniques. 1 . the total intra-operative blood loss $(\mathrm{mL}), 2$. the level of post-operative pain measured with the Visual Analog Scale (VAS) raging from 0 (no pain) to 10 (severe pain), 3. global, emotional, and physical post-operative states assessed with the standardized MDADI (M.D. Anderson Dysphagia Inventory) and psychosocial distress (PD) questionnaire.

Blood losses $(\mathrm{mL})$ were measured in the suction container (without the dilution with water) postoperatively. Additionally, after OS dry and used cotton swabs were weighed. After TORS, the amount of blood accumulated both in the stomach and naso-gastric feeding tube (NGFT) was quantified. The neck dissections associated blood losses were measured separately and subtracted from the total retained volume.

The MDADI (MD Anderson Dysphagia Inventory) questionnaire is a 20-item evaluation of swallowing-related quality of life ranging from 0 to 100 with higher scores indicating better perception of swallowing ${ }^{16}$.

Assessment of psychological distress (PD) includes eight of following items: physical functioning, bodily pain, role limitations due to physical health problems, role limitations due to personal or emotional problems, emotional well-being, social functioning, energy/fatigue and general health perceptions ${ }^{16}$. Each item was measured using a scale ranging from 0 to 10 . Definitive PD score was determined as the summation of particular values, correlating inversely with psychological status. All questionnaires were validated for Czech language ${ }^{17-19}$.

MDADI, VAS and PD scores were evaluated in intervals of 1 week, 6 weeks and 6 months after the surgery.

The differences between both tested groups were based at significance level $\alpha=0.05$ (for multiple comparisons) of Fisher's Least Significant Difference (LSD) test. 


\section{RESULTS}

\section{Blood loss}

Mean general OS associated blood loss was 405 (range from 275 to 520) $\mathrm{mL}$, that associated with lateral pharyngotomy and supraglottic laryngectomy was 495 (428-520) $\mathrm{mL}$ and 345 (275-394) $\mathrm{mL}$, respectively. The mean blood loss in TORS patients was 29 (14-45) $\mathrm{mL}$, in those with tongue base and supraglottic tumours 31 (18-25) $\mathrm{mL}$ and 27 (17-48) $\mathrm{mL}$, respectively (Fig. 1.). The statistical difference of the mean blood loss between OS and TORS procedure was highly significant $(\alpha=0.05)$ (Table 1.).

Global, emotional, functional, and physical difficulties related to surgery techniques and post-operation states (MDADI).

MDADI scores in all TORS patients recorded one week, six weeks and six months postoperatively ranged from 58 to 62 (mean 60.01), from 88 to 94 (mean 91.01) and from 93 to 95.5 (mean 94.18), respectively. OS associated MDADI values in the same time intervals ranged from 43 to 47 (mean 44.93), from 61 to 64 (mean 62.19) and from 92.6 to 94.5 (mean 93.56) (Fig. 2.). The mean MDADI score in TORS patients was significantly higher than that in OS patients one week and six weeks, but not six months postoperatively. Table 2 . shows the statistical difference of the MDADI between OS and TORS procedure.

\section{Levels of post-operation pain and related somatic difficulties (VAS)}

TORS associated VAS values measured one week, six weeks and 6 months after the procedure varied from 4.75 to 5.4 (mean 5.09), 1.85 to 2.4 (mean 2.09) and 1.15 to 1.41 (mean 1.27), respectively. OS associated one week, six week and 6 month VAS values ranged from 5.25 to 5.85 (mean 5.56), 2.8 to 3.3 (mean 3.11 ) and 1.22 to 1.45 (mean 1.33), respectively (Fig. 3.). Differences between both one week and six week, but not 6 month TORS and OS mean VAS values were significant. Table 3 . shows the statistical difference of the VAS between OS and TORS procedure.

\section{Psychosocial distress (PD)}

One week, six week and 6 month TORS associated PD values ranged from 25.2 to 28.8 (mean 26.82), 38.3 to 41.9 (mean 39.95 ), 43.7 to 45.8 (mean 44.73), respectively. The correspondent OS associated PD values varied from 23.4 to 26.8 (mean 25.11), 27.5 to 31.2 (mean 29.22) and 43.6 to 45.45 (mean 44.52), respectively (Fig. 4.). For all three time intervals, TORS associated mean PD values were higher than those associated with OS. However, only six week PD values bore statistically significant differences (Table 4.).

\section{DISCUSSION}

TORS and conventional OS represent two diverse options for surgical intervention in T1 - T2 carcinomas located at the tongue base or supraglottis ${ }^{20,21}$. Whereas the
Table 1. Blood loss - statistical difference between tested groups.

\begin{tabular}{cccc}
\hline Contrast & Sig. & Difference & $+/$ - Limits \\
\hline TORS - OS & $*$ & -378.759 & 24.964 \\
\hline
\end{tabular}

*Statistical significance at $\alpha=0.05$.

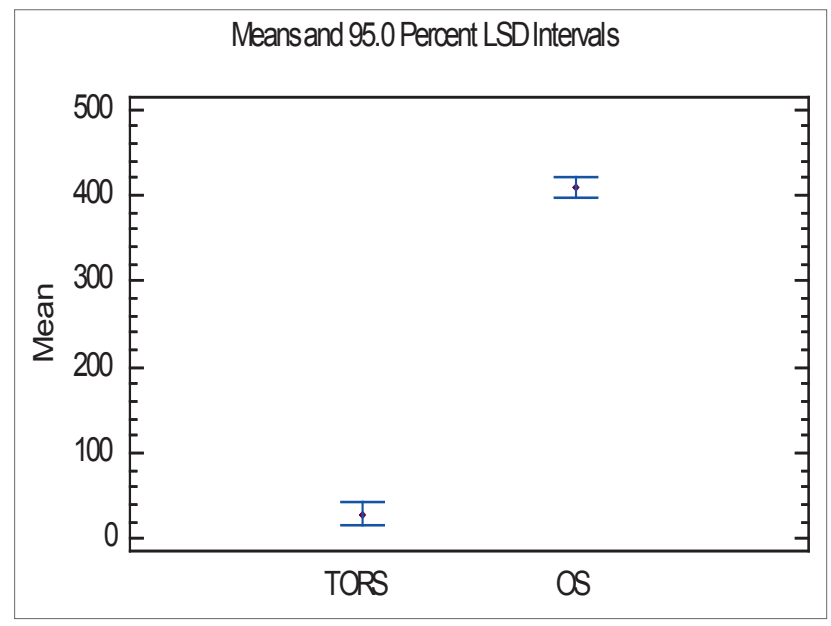

Fig. 1. Blood losses in TORS and OS $(\mathrm{mL})$.

most suitable tumours for the former procedure are considered those placed on or slightly below the mucosa of concerned anatomical regions, the latter is being applied in more deeply situated lesions and/or in patients showing limited transoral access to hypopharynx ${ }^{22}$. In contrast to OS, TORS is associated with substantially minor invasiveness suggesting modest blood loss, limited postoperative pain as well as swallowing and psychosocial distress. To the best of our knowledge, relevant comparison has not been published in the English literature so far.

In our series, the average OS associated blood loss outnumbered significantly that measured in TORS patients, which was $29.0 \mathrm{~mL}$. Such a very low value in the latter group meets those reported by Weinstein in a multicenter study dealing with surgical aspects of robotic surgery ${ }^{23}$. In the two involved institutions (Mayo Clinic, Rochester and University of Alabama, Birmingham), the average volumes were $30.2 \mathrm{~mL}$ and $37.2 \mathrm{~mL}$, respectively. However, considerably higher values reaching $120.5 \mathrm{~mL}$ were reported from University of Pennsylvania (Philadelphia). Unequivocal explanation for such a great inter-institutional difference can be seen in extensive parapharyngeal space TORS procedures performed at the latter university.

Significant differences in 1 and 6 week MDADI scores between TORS and OS patients document much less intensive swallowing difficulties in the former group. This is conceivable considering only slight oedema of and heamorrhage to the neck soft tissues caused by robotic surgery. As anticipated, there was no essential distinction in MDADI scores between TORS and OS group 6 months postoperatively. During that time complete regression of initially extensive tissue damage associated with the latter surgery occurred. 
Table 2. MDADI - statistical difference between tested groups

\begin{tabular}{lclc}
\hline Contrast & Sig. & Difference & +/- Limits \\
\hline $\begin{array}{l}\text { MDADI } \\
1 \text { week TORS - OS }\end{array}$ & $*$ & 15.165 & 3.4086 \\
$\begin{array}{l}\text { MDADI } \\
6 \text { weeks TORS - OS }\end{array}$ & $*$ & 28.9057 & 3.5597 \\
$\begin{array}{l}\text { MDADI } \\
6 \text { months TORS - OS }\end{array}$ & & 0.626263 & 2.42509 \\
\hline
\end{tabular}

* Statistical significance at $\alpha=0.05$.

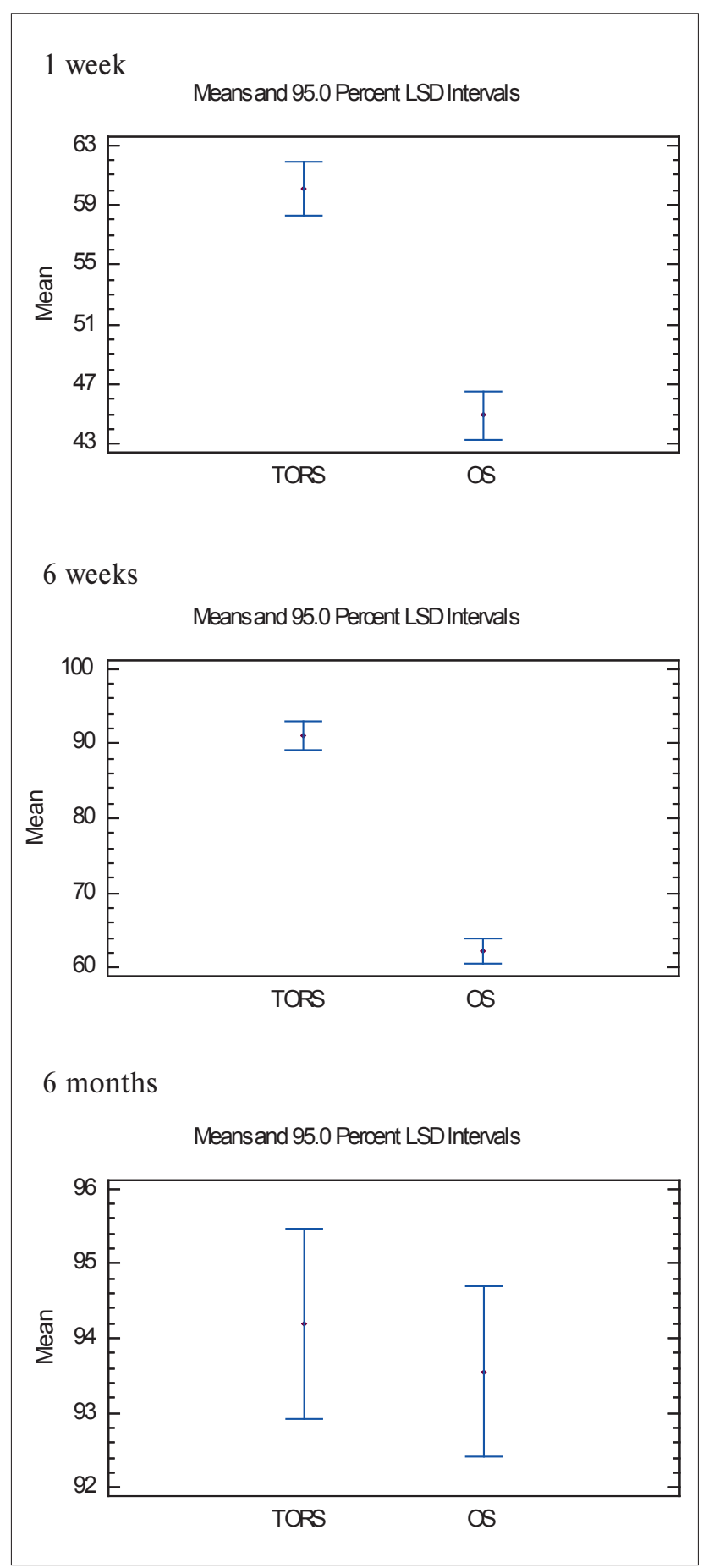

Fig. 2. MDADI scores in the selected intervals TORS and OS.
Table 3. VAS - statistical difference between tested groups.

\begin{tabular}{lclc}
\hline Contrast & Sig. & Difference & +/- Limits \\
\hline $\begin{array}{l}\text { VAS } \\
1 \text { week TORS - OS }\end{array}$ & $*$ & -0.464646 & 0.622109 \\
$\begin{array}{l}\text { VAS } \\
6 \text { weeks TORS - OS }\end{array}$ & $*$ & -1.0202 & 0.480363 \\
$\begin{array}{l}\text { VAS } \\
6 \text { months TORS - OS }\end{array}$ & & -0.0606061 & 0.271322 \\
\hline
\end{tabular}

* Statistical significance at $\alpha=0.05$.

1 week

Meansand 95.0 Percent LSD Intervals

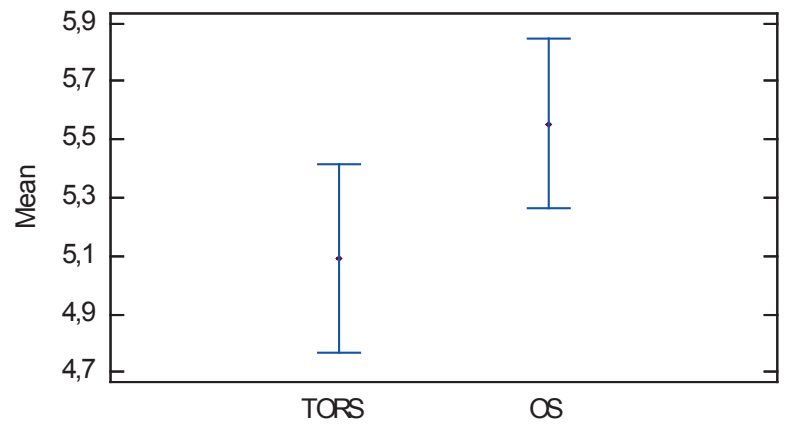

6 weeks

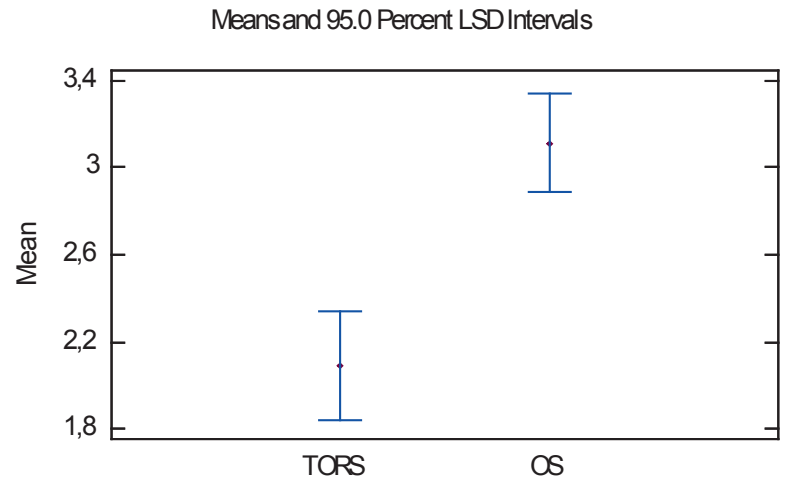

6 months

Meansand 95. 0 Percent LSD Intervals

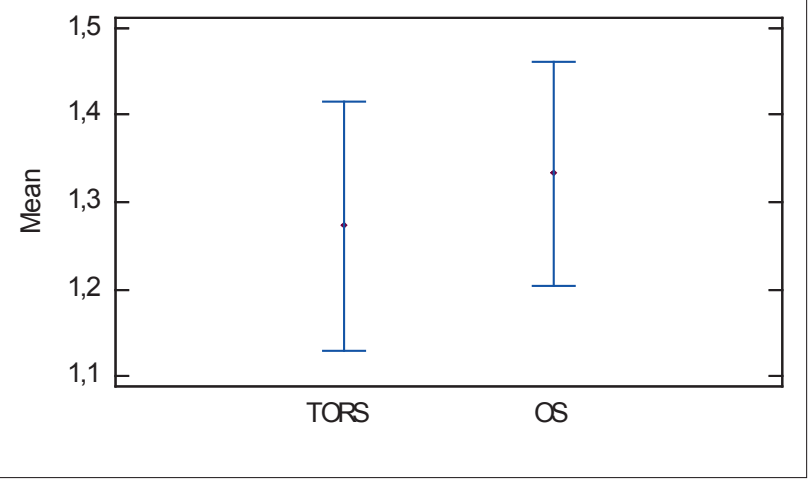

Fig. 3. VAS scores in the selected intervals in TORS and OS. 
Table 4. PD - statistical difference between tested groups.

\begin{tabular}{lccc}
\hline Contrast & Sig. & Difference & +/- Limits \\
\hline PD & & 1.70707 & 3.5717 \\
1 week TORS - OS & $*$ & 10.7323 & 3.54543 \\
PD & & & \\
6 weeks TORS - OS & & 0.208754 & 1.98571 \\
PD & & & \\
6 months TORS - OS & &
\end{tabular}

* Statistical significance at $\alpha=0.05$

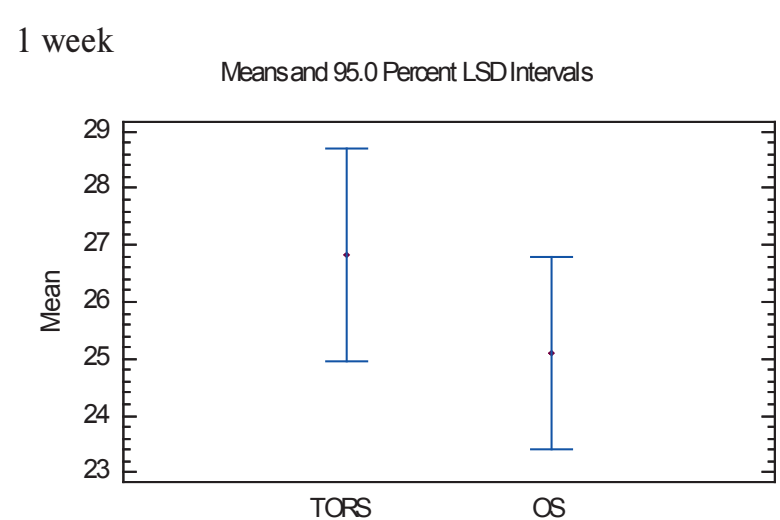

6 weeks Meansand 95.0 Perœent LSDIntervals

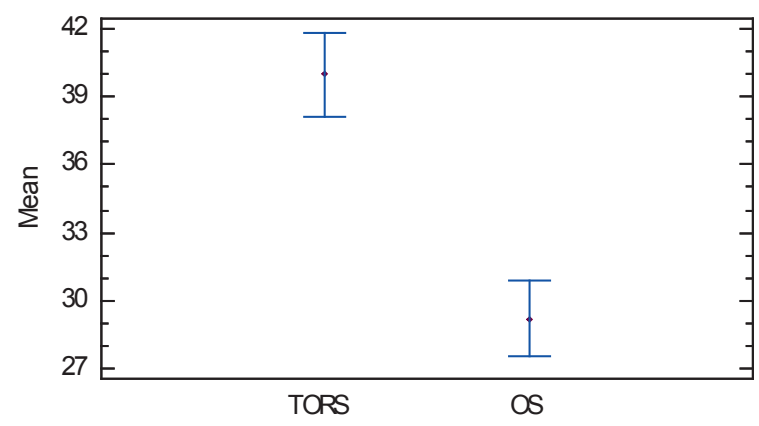

6 months

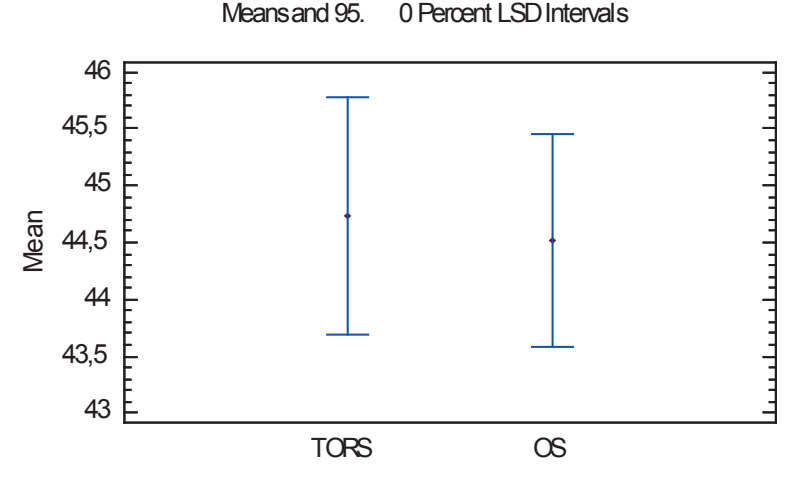

Fig. 4. PD scores in the selected intervals in TORS and OS.
Modest TORS associated soft tissue trauma was also confirmed by Mercante ${ }^{24}$. Using a three scale score, this author assessed dysphagia in 13 patients diagnosed with $\mathrm{T} 1$ or T2 tumours originating from the base of the tongue. One week postoperatively, nine, and two each of them indicated no, minor and major swallowing difficulties, respectively. Such an early return to normal deglutition correlates with the results of MDADI in our study.

Noticeable differences between the perception of TORS and OS associated pain were recorded 1 and 6 weeks, but not 6 months postoperatively, which paralleled those in swallowing difficulties associated with particular types of surgery. This analogy accrues from practically identical mechanisms involved in both tested complaints, i.e. heamorrhage and oedema. In swallowing difficulties a functional component resulting from impairment of pharyngeal constrictor muscle that is inavoidable in open surgery, may also play a role. Six months postoperatively, full activity of this muscle returned.

Significant distinction in PD scores between the two assessed surgical methods occured exclusively six weeks postoperatively. At the same time, marked contrast in the intensity of pain between TORS and OS patients was also present. However, such chronological correlation between PD and pain was absent at 1 week interval. Plausible explanation might be seen in that $\mathrm{PD}$ represents reverse category of wellbeing, in the evaluation of which perception of pain is but one of many highly subjective factors. At that time, the latter probably overwhelmed gradually fading physical deprivation.

In comparison to OS, TORS was not associated with higher locoregional recurrence rate, both methods thus show comparable oncological safety.

\section{CONCLUSION}

This study revealed that TORS associated blood loss, pain and swallowing difficulties were significantly lower than those in OS in the short post-op period. The methods were comparable in terms of locoregional tumour recurrence. This confirms our assumption of the former surgery as a minimally invasive procedure which positively influences healing and reduces psychosocial distress in patients with carcinomas of the tongue base and supraglottis.

Author contributions: KS: manuscript writing and revising, leader and coordinator of study, TORS and OS procedures performed at Masaryk Hospital in Usti nad Labem, literature search, final approval; DS, JS: manuscript writing and revising, OS procedures performed at Faculty of Medicine in Pilsen, final approval; SF: manuscript writing and revising, psychosocial distress research, statistical tests, final approval.

Conflict of interest statement: The authors state that there are no conflicts of interest regarding the publication of this article. 


\section{REFERENCES}

1. Lefebvre JL, Vermorken JB. Epidemiology overview of squamous cell carcinoma of the head and neck. Springer 2006;3:2-7.

2. Ang KK, Harris J, Wheeler R, Weber R, Rosenthal DI, Nguyen-Tân PF, Westra WH, Chung CH, Jordan RC, Lu C, Kim H, Axelrod R, Silverman CC, Redmond KP, Gillison ML. Human papillomavirus and survival of patients with oropharyngeal cancer. N Engl J Med 2010;363(1):24-35.

3. Chaturvedi AK, Engels EA, Pfeiffer RM, Hernandez BY, Xiao W, Kim E, Jiang B, Goodman MT, Sibug-Saber M, Cozen W, Liu L, Lynch CF, Wentzensen N, Jordan RC, Altekruse S, Anderson WF, Rosenberg PS, Gillison ML. Human papillomavirus and rising oropharyngeal cancer incidence in the United States. J Clin Oncol 2011;29(32):4294-301.

4. Peretti G, Piazza C, Cocco D. Transoral CO2 laser treatment for Tis-T3 glottic cancer: the university of Brescia experience of 595 patients. Head Neck 2010;32:977-83.

5. Hinni ML, Salassa JR, Grant DG, Pearson BW, Hayden RE, Martin A Christiansen $\mathrm{H}$, Haughey BH, Nussenbaum B, Steiner W. Transora laser microsurgery for advanced laryngeal cancer. Arch Otolaryngo Head Neck Surg 2007;133:1198-204

6. Aubry K, Yachine M, Perez AF, Vivent M, Lerat J, Scomparin A, Bessède JP. Transoral robotic surgery for head and neck cancer: a series of 17 cases. Eur Ann Otorhinolaryngol Head Neck Dis 2011; 28(6):290-6.

7. Weinstein GS, O'Malley BW Jr, Cohen MA, Quon H. Transoral robotic surgery for advanced oropharyngeal carcinoma. Arch Otolaryngol Head Neck Surg 2010;136(11):1079-85.

8. Park YM, Kim WS, Byeom HK, Lee SY, Kim SH. Surgical techniques and treatment outcomes of transoral robotic supraglottic partia laryngectomy. Laryngoscope 2013;123(3):670-7.

9. O'Malley BW Jr, Weinstein GS, Snyder W, Hockstein NG. Transoral robotic surgery (TORS) for base of tongue neoplasms. Laryngoscope 2006;116(8):1465-72.

10. Moore EJ, Olsen SM, Laborde RR, García JJ, Walsh FJ, Price DL, Janus JR, Kasperbauer JL, Olsen KD. Long-term functional and oncologic results of transoral robotic surgery for oropharyngeal squamous cell carcioma. Mayo Clin Proc 2012;87(3):219-25.

11. Moore EJ, Olsen KD, Kasperbauer JL. Transoral robotic surgery for oropharyngeal squamous cell carcinoma: a prospective study of feasibility and functional outcomes. Laryngoscope 2009;119(11):215664.

12. Weinstein GS, O'Malley BW Jr, Snyder W, Hockstein NG. Transora robotic surgery: supraglottic partial laryngectomy. Ann Oto Rhino Laryngol 2007;116(1):19-23.
13. Sobin LH, Fleming ID. TNM classification of malignant tumors. Cancer 1997;80(9):1803-4

14. Laurie AL, Kim OL, Suyash Mohan, O'Malley BW Jr, Mary H.Scalon, Christopher HR, Weinstein GS. Transoral robotic surgery in Head and Neck Cancer: What radiologists need to know about the cutting edge. Radiographics 2013;33:1764-72.

15. Bernier J, Cooper JS, Pajak TF, van Glabbeke M, Bourhis J, Forastiere A, Ozsahin EM, Jacobs JR, Jassem J, Ang KK, Lefèbvre JL. Defining risk levels in locally advanced head and neck cancers: a comparative analysis of concurrent postoperative radiation plus chemotherapy trials of the EORTC nad RTOG. Head Neck 2005;27(10):843-50.

16. Chen AY, Frankowski R, Bishop-Leone J, Herbert T, Leyk S, Lewin J, Goepfert $\mathrm{H}$. The development and validation of dysfagia-specific quality-of-life qustionaire for patients with head and neck cancer: the M.D. Anderson dysphagia inventory. Arch Otolaryngol Head Neck Surg 2001;127(7):870.

17. Mandysová P, Petržílková K, Škvrňáková J, Ehler E. An analysis of subjective assessment of swallowing function in older adults using the eat-10 questionaire. Central European Journal of Nursing and Midwifery 2014;5(2):72-7

18. Vymětal J. Lékařská psychologie. Praha: Portál 2003; ISBN 80-7178$40-X$.

19. Svoboda M. Psychologická diagnostika dospělých, Praha: Portál 2010; ISBN 978-80-7367-706-0.

20. O'Malley BW, Weinstein GS, Snyder W, Hockstein NG. Transoral robotic surgery (TORS) for base of tongue neoplasm. Laryngoscope 2006;116(8):1465-72.

21. Eisen MD, Weinstein GS, Chalian A, Chalian A, Machtay M, Kent K, Coia LR, Weber RS. Morbidity after midline mandibulotomy and radiation therapy. Am J Otolaryngol 2000;21(5):312-17.

22. Iseli TA, Kulbersh BD, Iseli CE, Carroll WR, Rosenthal EL, Magnuson JS. Functional outcomes after transoral robotic surgery for head and neck cancer. Otolaryngol Head Neck Surg 2009;141(2):166-71.

23. Weistein GS, O'Malley BW Jr, Magnuson JS, Carroll WR, Olsen KD, Daio L, Moore EJ, Holsinger FC. Transoral robotic surgery: a multicenter study to assess feasibility, safety, and surgical margins. Laryngoscope 2012;122(8):1701-7.

24. Mercante G, Ruscito P, Pellini R, Cristallini G, Spriano G. Transoral robotic surgery(TORS) for tongue base tumours. Acta otorhinolaryngologica italica 2013;33:230-5. 